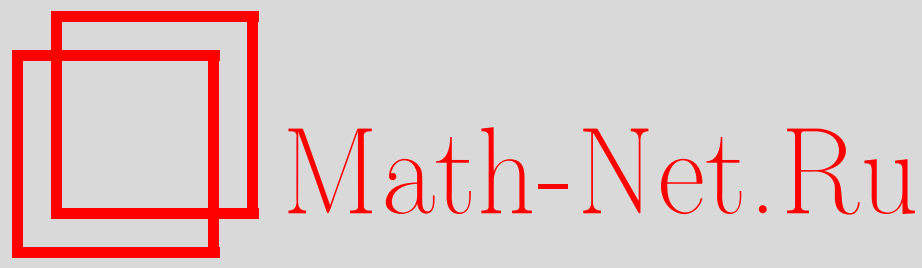

T. К. Ребане, Границы энергии системы гравитирующих бозонов, ТМФ, 2010, том 162, номер 3, 416-421

DOI: https://doi.org/10.4213/tmf6479

Использование Общероссийского математического портала Math-Net.Ru подразумевает, что вы прочитали и согласны с пользовательским соглашением http://www . mathnet.ru/rus/agreement

Параметры загрузки:

IP : 54.162 .127 .20

26 апреля 2023 г., 13:40:55

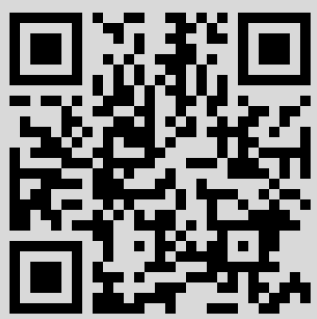




\title{
ФИЗИКА
}

Том 162, № 3

март, 2010

2010 г.

\section{ГРАНИЦЫ ЭНЕРГИИ СИСТЕМЫ ГРАВИТИРУЮЩИХ БОЗОНОВ}

\begin{abstract}
Методом, свободным от расчетов волновых функций, вычислена нижняя граница энергии системы взаимно гравитирующих бозонов с любым числом частиц $n$. Метод основан на использовании геометрических свойств треугольников, образуемых частицами. Найдены пределы коэффициента $C$ в асимптотике нерелятивистской энергии основного состояния гравитационной системы.
\end{abstract}

Ключевые слова: квантовая механика, границы энергии, бозоны, гравитация.

\section{1. ВВЕДЕНИЕ}

Квантово-механические системы одинаковых бозонов, между которыми действуют силы притяжения, представляют интерес в связи с фундаментальными проблемами устойчивости материи [1]-[3] и астрофизики [4]-[6], а также с моделированием взаимодействия бозе-эйнштейновских конденсатов [7].

$\mathrm{K}$ настоящему времени выполнены расчеты многобозонной гравитационной и родственной ей системы со взаимодействием Юкавы по методу самосогласованного поля [5], [6], а также многопараметрические вариационные расчеты трех- и четырехбозонных гравитационных систем [7], [8]. Эти результаты представляют собой верхние границы нерелятивистской энергии.

В настоящей работе определяется нижняя граница энергии гравитационной системы $n$ одинаковых бозонов. Используется разработанный автором метод, основанный на сочетании модельного уравнения Шредингера с элементарными геометрическими неравенствами, который не требует никаких расчетов волновых функций исследуемых систем.

\section{2. ОСНОВНЫЕ СООТНОШЕНИЯ}

Оператор энергии нерелятивистской $n$-бозонной гравитационной системы имеет Вид

$$
H=-\hbar^{2} \sum_{j=1}^{n} \frac{\Delta_{j}}{2 m}-G m^{2} \sum_{j<k}^{n} \frac{1}{r_{j k}} ;
$$

* Научно-исследовательский институт физики им. В. А. Фока Санкт-Петербургского государственного университета, Санкт-Петербург, Россия. E-mail: trebane@mail.ru 
его низшее собственное значение и соответствующая собственная функция определяются уравнением

$$
H \Psi=E \Psi .
$$

Составим волновую функцию, экспоненциально зависящую от всех расстояний между частицами:

$$
\Phi=\exp \left(-G m^{3} \sum_{j<k}^{n} \frac{r_{j k}}{2 \hbar}\right) .
$$

Эта функция не имеет узлов и удовлетворяет следующему уравнению Шредингера:

$$
\left[-\hbar^{2} \sum_{j=1}^{n} \frac{\Delta_{j}}{2 m}-G m^{2} \sum_{j<k}^{n} \frac{1}{r_{j k}}+G^{2} m^{5} \sum_{j \neq k, k<l}^{n} \frac{\cos \theta_{j k l}}{4 \hbar^{2}}\right] \Phi=E_{\bmod } \Phi .
$$

Она является собственной функцией основного состояния модельной системы, описываемой оператором энергии вида

$$
H_{\text {mod }}=-\hbar^{2} \sum_{j=1}^{n} \frac{\Delta_{j}}{2 m}-G m^{2} \sum_{j<k}^{n} \frac{1}{r_{j k}}+G^{2} m^{5} \sum_{j \neq k, k<l}^{n} \frac{\cos \theta_{j k l}}{4 \hbar^{2}} .
$$

Через $\theta_{j k l}$ обозначен угол с вершиной у частицы $j$, стороны которого связывают ее с частицами $k$ и $l ; \cos \theta_{j k l}=\left(\mathbf{r}_{\mathbf{k}}-\mathbf{r}_{\mathbf{j}}, \mathbf{r}_{\mathbf{l}}-\mathbf{r}_{\mathbf{j}}\right) /\left(r_{j k} r_{j l}\right)$. Энергия основного состояния модельной системы равна

$$
E_{\mathrm{mod}}=-n(n-1) \frac{G^{2} m^{5} / \hbar^{2}}{8 \hbar^{2}}
$$

Операторы энергии реальной и модельной систем связаны равенством

$$
H=H_{\bmod }-W
$$

где оператор $W$ определяется “мгновенным" расположением частиц:

$$
W=\sum_{j \neq k, k<l} \cos \theta_{j k l} \frac{G^{2} m^{5}}{4 \hbar^{2}} .
$$

Из уравнений (2) и (7) и вариационного принципа для энергии основного состояния модельной системы $H_{\text {mod }}$ следует цепочка соотношений

$$
E=\langle\Psi|H| \Psi\rangle=\left\langle\Psi\left|H_{\mathrm{mod}}-W\right| \Psi\right\rangle \geqslant E_{\mathrm{mod}}-\langle\Psi|W| \Psi\rangle,
$$

определяющая нижнюю границу энергии гравитационной системы:

$$
E \geqslant-\left[n(n-1)+2\left\langle\Psi\left|S_{n}\right| \Psi\right\rangle\right] \frac{G^{2} m^{5}}{8 \hbar^{2}},
$$

где $S_{n}$ - сумма "мгновенных" значений косинусов углов $\theta_{j k l}$ :

$$
S_{n}=\sum_{j \neq k, k<l}^{n} \cos \theta_{j k l}
$$


Так как $\left|\cos \theta_{j k l}\right| \leqslant 1$, то эта сумма имеет конечное значение при всех возможных вариантах расположения частиц. Ее квантово-механическое среднее значение не превосходит ее максимума относительно расположений $n$ точек в трехмерном пространстве:

$$
\left\langle\Psi\left|S_{n}\right| \Psi\right\rangle \leqslant \max S_{n}
$$

\section{3. ГРАНИЦЫ ЭНЕРГИИ}

Из формулы (10) для нижней границы энергии получим

$$
E \geqslant-\frac{\left[n(n-1)+2 \max S_{n}\right] G^{2} m^{5}}{8 \hbar^{2}} .
$$

Достойно внимания то, что с помощью этого неравенства оценка энергии квантово-механической системы сводится к геометрической задаче- отысканию максимума суммы косинусов углов, образуемых частицами.

Неравенство (13) остается в силе, если заменить величину $\max S_{n}$ ее оценкой сверху. Из неравенства $\left|\cos \theta_{j k l}\right| \leqslant 1$ получим грубую оценку $\max S_{n} \leqslant n(n-1)(n-2) / 2$. Ее можно улучшить, представив сумму $S_{n}$ как сумму вкладов $n(n-1)(n-2) / 6$ треугольников, образуемых $n$ частицами. Сумма косинусов внутренних углов треугольника лежит в пределах от 1 до $3 / 2$ (первый предел соответствует вырождению треугольника в отрезок прямой, второй - равностороннему треугольнику). Отсюда следует оценка

$$
\max S_{n} \leqslant \frac{n(n-1)(n-2)}{4},
$$

и из формулы (13) получим для энергии простую нижнюю границу:

$$
E \geqslant-\frac{n^{2}(n-1) G^{2} m^{5}}{16 \hbar^{2}}
$$

Неравенство (14) для $\max S_{n}$ переходит в равенство только тогда, когда возможна конфигурация $n$ точек, в которой все образуемые ими треугольники равносторонние. Это возможно при $n=3$ или $n=4$. При $n \geqslant 5$ таких конфигураций не существует. В этом случае для повышения точности нижней границы энергии требуются непосредственные расчеты максимума суммы $S_{n}$.

На рис. 1 представлены рассчитанные при различных $n$ максимальные средние значения косинусов углов

$$
\max (\cos \theta)_{n}=\frac{2 \max S_{n}}{n(n-1)(n-2)} .
$$

Нижняя граница энергии (13) определяется величиной $\max (\cos \theta)_{n}$ :

$$
E \geqslant-n(n-1)(n-2) \frac{\left[\max (\cos \theta)_{n}+1 /(n-2)\right] G^{2} m^{5}}{8 \hbar^{2}} .
$$

C ростом числа частиц $n$ величина $\max (\cos \theta)_{n}$ убывает от значения $1 / 2$ до предельного значения $4 / 9$ (при $n \rightarrow \infty)$ ). Последнее соответствует равномерному распределению частиц по поверхности сферы. Одновременно с этим происходит повышение (т.е. уточнение) нижней границы энергии (17) по сравнению с простой границей (15). 


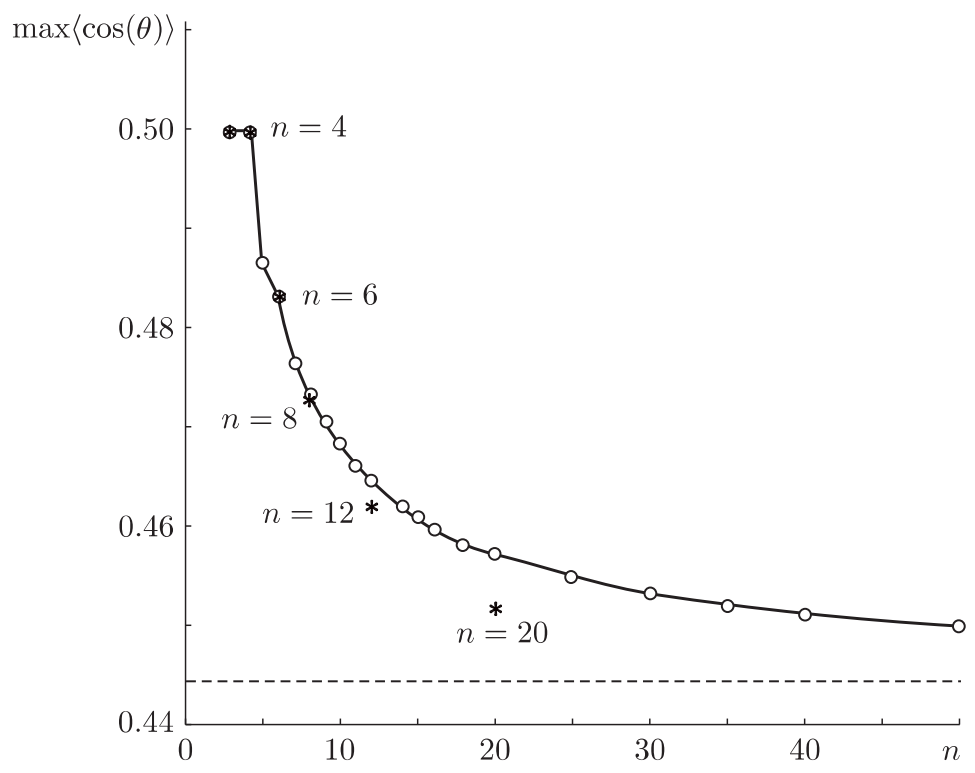

Рис. 1. Максимальное среднее значение косинусов углов, образуемых $n$ частицами. Сплошная линия - численная оптимизация геометрической конфигурации частиц, звездочки - случаи расположения частиц в вершинах правильных многогранников. Штриховая линия - асимптота $\max \langle\cos \theta\rangle_{n}=4 / 9$ при $n \rightarrow \infty$.

На рис. 2 сопоставляются три границы энергии: простая нижняя граница (15) (линия 1), уточненная нижняя граница (17) (линия 2) с асимптотой (линия 3) и верхняя граница, рассчитанная по методу самосогласованного поля (линия 4) [5] при отделении движения центра масс:

$$
E(n) \leqslant-\frac{0.05426 n^{2}(n-1) G^{2} m^{5}}{\hbar^{2}} .
$$

Эти границы имеют при больших $n$ асимптотику $E \sim-C n^{3} G^{2} m^{5} / \hbar^{2}$, где соответственно $C=-0.0625,-0.05555$ и -0.05426 . Первое из них было найдено также в работе [9] способом редукции многочастичной задачи к двухчастичной. Таким образом, неизвестное точное значение коэффициента лежит в пределах $-0.05555 \leqslant$ $C \leqslant-0.05426$. Расстояние между уточненной нижней границей (17) и самосогласованной верхней границей (18) убывает с ростом числа частиц в пределе до $2.5 \%$.

Точные значения нерелятивистской энергии при всех $n$ заключены между этими границами.

Теорема вириала связывает среднее значение обратных междучастичных расстояний с энергией системы:

$$
-\frac{n(n-1) G m^{2}\langle 1 / r\rangle}{2}=2 E(n)=-\frac{2 C n^{2}(n-1) G^{2} m^{5}}{\hbar^{2}} .
$$




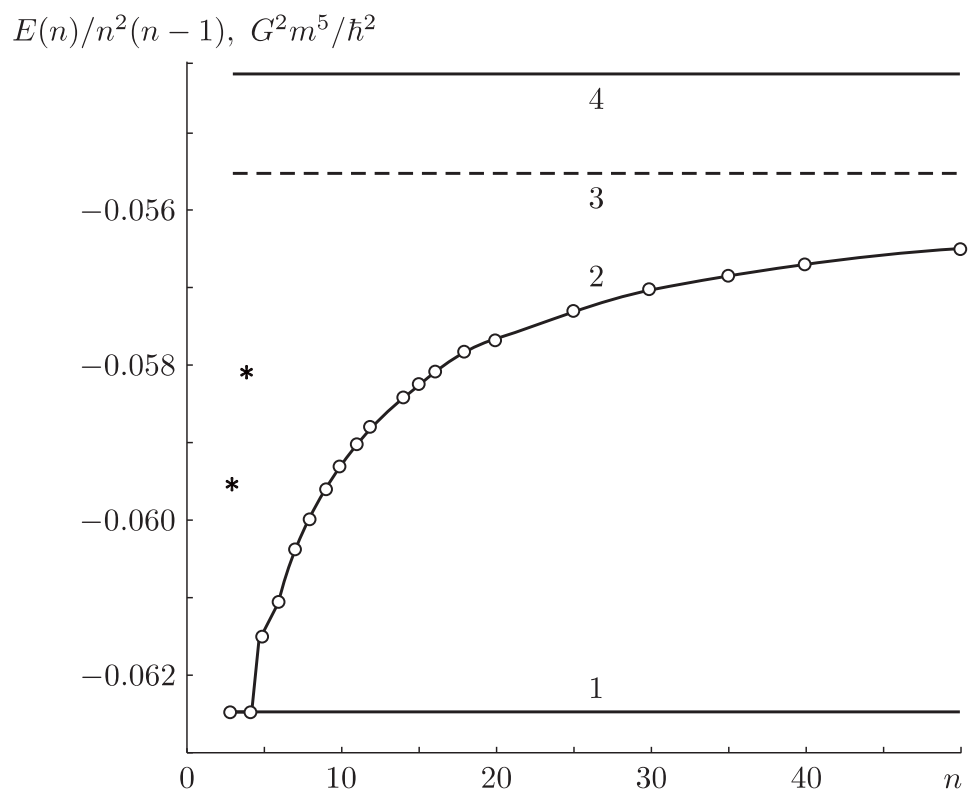

Рис. 2. Границы приведенной энергии гравитационной системы $n$ бозонов. Звездочками отмечены результаты вариационных расчетов при $n=3$ [7] и $n=4[8]$.

Для среднего расстояния между частицами получим оценку

$$
\langle r\rangle \sim \frac{1}{\langle 1 / r\rangle}=\frac{\hbar^{2}}{4 C G m^{3} n},
$$

а плотность частиц пропорциональна $n^{3}$.

При больших $n$ это приводит к гравитационному коллапсу, для описания которого необходим учет релятивистских эффектов. Поэтому физические выводы из полученных здесь результатов относятся к разреженным системам частиц, плотность которых далека от гравитационного коллапса.

\section{4. ЗАКЛЮЧЕНИЕ}

Предложенный здесь “геометрический” подход позволяет заключить энергии нерелятивистских гравитационных систем в узкие границы, но существенным является ограниченность “возмущения" $W$, т.е. отклонения модельного гамильтониана от реального. Благодаря корректному поведению модельной волновой функции $\Phi$ при малых междучастичных расстояниях, он свободен от сингулярностей типа $1 / r_{j k}$. Оператор $W$ остается ограниченным и для кулоновских систем при использовании модельной волновой функции

$$
\Phi=\exp \left[-\sum_{j<k}^{n} \frac{q_{j} q_{k} m_{j} m_{k} r_{j k}}{\left(m_{j}+m_{k}\right) \hbar}\right]
$$


где $q_{j}$ и $m_{j}$ - заряды и массы частиц. Это позволяет применять изложенный здесь подход также и к кулоновским системам, в которых сочетаются силы притяжения и отталкивания.

\section{Список литературы}

[1] F. J. Dyson, A. Lenard, J. Math. Phys., 8:3 (1967), 423-434.

[2] E. H. Lieb, Rev. Modern Phys., 48:4 (1976), 553-569.

[3] E.H. Lieb, The Stability of Matter: From Atoms to Stars, Selecta of Elliott H. Lieb, ed. W.E. Thirring, Springer, Berlin, 2001.

[4] R. Ruffini, S. Bonazzola, Phys. Rev., 187:5 (1969), 1767-1783.

[5] M. Membrado, A. F. Pacheco, J. Sañudo, Phys. Rev. A, 39:8 (1989), 4207-4211.

[6] R. L. Hall, Phys. Rev. A, 45:11 (1992), 7682-7687.

[7] S. Kar, Y.K. Ho, Phys. Lett. A, 370:3-4 (2007), 306-309.

[8] Т. К. Ребане, О.Н. Юсупов, ЖЭТФ, 102:6 (1992), 1762-1767.

[9] H. R. Post, Proc. Phys. Soc., 79 (1962), 819-820.

Поступила в редакцию 6.07.2009 